\title{
THERMODYNAMICS OF ADSORPTION AND GIBBSIAN DISTANCE PARAMETERS IN TWO- AND THREE-PHASE SYSTEMS
}

\author{
ROBERT J. GOOD \\ Department of Chemical Engineering, State University of New York at Buffalo, Buffalo, NY 14214, USA
}

Abstract-In a multicomponent, 2-phase system, the Gibbs dividing surfaces for the respective components are separated by characteristic distances,

$$
\lambda_{i j}=\Gamma_{j}^{(i)} / \Delta c_{j}
$$

More generally, if $b$ designates an arbitrary criterion defining a surface, e.g. $\Gamma_{i}=\Gamma_{i}$, and $d$ designates another criterion, e.g. the surface of tension,

$$
\lambda_{b d}=\left(\Gamma_{i}^{(b)}-\Gamma_{i}^{(d)}\right) / \Delta c_{i}=\left(\Gamma_{j}^{(b)}-\Gamma_{j}^{(d)}\right) / \Delta c_{j} .
$$

For a binary, liquid-liquid system,

$$
\lambda_{i j}=\left(\frac{\partial r}{\partial p}\right)_{T, \text { sat }}
$$

In a 3-phase system with a line of 3-phase contact, it is possible to set up the one-dimensional analog of the Gibbs dividing surface, and to treat a linear excess mass that corresponds to the Gibbsian surface excess mass. The line of tension is the analog of the surface of tension. A linear thermodynamics can be developed, that is the one-dimensional analog of Gibbsian surface thermodynamics. Applications of eqns (I and III) are reported, for the estimation of $\lambda$. For the interface between air and solutions of water and ethanol, at mole fraction ethanol $=0.18, \lambda_{12}$ is $8.8 \AA$. For the benzene-water system, $\lambda_{12}$ is found to be about $0.1 \AA$.

\section{INTRODUCTION}

The spatial location of Gibbsian dividing surfaces in a binary or ternary system has received little attention in the past. Gibbs 1 conjectured that the " $\Gamma=0$ " surface was at an experimentally negligible distance from the surface of tension. Buff and co-workers ${ }^{2-4}$ have treated the distance between the $\Gamma=0$ surface, in a one-component system, and those surfaces with respect to which the surface excess energy, $U^{\sigma}$, and entropy, $S^{\sigma}$, are zero.

Guggenheim and Adam $^{5}$ have concluded that the superficial excess of matter, in a binary liquid-vapor system, is dependent on the convention in which concentrations in the surface region are expressed for the purpose of defining the reference dividing surface. This dependence on conventions may have discouraged theoretical investigation, in this field. We will show, below, that this defect of convention can be removed.

Nomenclature and notation are, all too often, not given their due, as ingredients in the advancement of thermodynamics. The common attitude of theoreticians is, to embody their own understanding of thermodynamics in notation which, if properly interpreted, says all that needs to be said. But their interpretations are not always accessible to subsequent workers, and so sometimes are lost. It may even be questioned whether the interpretations existed at all, or were clear, in the minds of the pioneers. Gibbsian surface thermodynamics is a case in point. If a notation could be introduced which made the interpretations clear, it might remove much of the well-known abstractness and lack of usefulness of surface thermodynamics. A part of the contribution of this paper will amount to a very simple change in notation, which clarifies the interpretation of certain concepts which Gibbs left obscure.

\section{THEORY: TWO-PHASE SYSTEMS}

As a preliminary, we note that in a binary, 2-phase system, the Phase Rule allows no variation of composition, at constant temperature and pressure. Hence, the general statement of the Gibbs adsorption isotherm equation is limited to systems with 3 or more components:

$$
-\left[\frac{\partial \gamma_{\alpha \beta}}{\partial \mu_{1}}\right]_{T, P}=\Gamma_{1}+\sum_{i=1}^{n} \Gamma_{i}\left[\frac{\partial \mu_{i}}{\partial \mu_{1}}\right]_{T, P}, \quad n \geqq 2 .
$$

Here $\gamma_{\alpha \beta}$ is the interfacial tension between phases $\alpha$ and $\beta, \mu$ is chemical potential, and $\Gamma$ is surface excess of matter.

For the same reason, the Gibbs-Duhem equation at constant temperatures and pressure (which is the form employed by most surface scientists) is limited to systems with three or more components:

$$
\left.\begin{array}{l}
\sum_{i=1}^{n} N_{i}^{\alpha} \mathrm{d} \mu_{i}=0 \\
\sum_{i=1}^{n} N_{i}^{\beta} \mathrm{d} \mu_{i}=0
\end{array}\right\} n \geqq 3
$$

where $N_{i}$ is the number of moles in $i$ in the designated phase. Defay et al. $^{6}$ have pointed out that measurements on ordinary 2-component, liquid-vapor systems are made in air or an "inert" gas, and if the component that comprises the gas phase is not appreciably adsorbed at the liquid-vapor interface, the ternary system may, as a good approximation, be treated as a binary with the Phase Rule relaxed.

For a binary, liquid-liquid system, this treatment, i.e. eqn (2) with $n=2$, cannot be employed. Guggenheim erred in this regard, ${ }^{7}$ and obtained an incorrect set of equations for liquid-liquid systems. 
Three-phase systems may exist, in which the number of components is one, or two, or three. We will be interested, here, in binary or ternary systems in which there is a non-zero contact angle $\theta$. At constant temperature and pressure, these are invariant. Hence, only if there are four or more components, can the Gibbs equation at constant $T$ and $P$ be employed.

Since the differential, $\mathrm{d} \mu_{i}$, and the derivative $\left(\partial \mu_{j} / \partial \mu_{i}\right)_{T, P}$ do not exist in an invariant system, we should not even write the differential form of the Gibbs equation for a binary, without noting that both $T$ and $P$ may not be kept constant. If, then, we allow $T$ or $P$ to vary, we are led to the conditions under which a Gibbsian treatment of adsorption can be carried out for a binary, liquid-liquid system: variation along the saturation line of the phase diagram, i.e. variation such that

$$
\mu_{i}^{\alpha}=\mu_{i}^{\beta}, \quad i=1 \text { or } 2 .
$$

We now consider the Gibbs equation, subject to these limitations.

$$
-\mathrm{d} \gamma_{\alpha \beta}=S^{\sigma} \mathrm{d} T+\sum_{i} \Gamma_{i} \mathrm{~d} \mu_{i} .
$$

We designate the saturation condition, i.e. eqn (3), by the subscript "sat." Then from eqn (4), for a binary, 2-phase system,

$$
\begin{gathered}
-\left[\frac{\partial \gamma_{\alpha \beta}}{\partial T}\right]_{P, \text { sat }}=S^{\sigma}+\sum_{i=1}^{2} \Gamma_{i}\left[\frac{\partial \mu_{i}}{\partial T}\right]_{P, \text { sat }} \\
-\left[\frac{\partial \gamma_{\alpha \beta}}{\partial P}\right]_{T, \text { sat }}=\sum_{i=1}^{2} \Gamma_{i}\left[\frac{\partial \mu_{i}}{\partial P}\right]_{T, \text { sat. }}
\end{gathered}
$$

We now undertake the problem of the locations of Gibbsian dividing surfaces relative to the plane that we intuitively recognize as the "physical" surface. Actually, the latter has a unique definition only for a molecularly smooth solid. And, even for the smoothest possible solid surface, the $\Gamma_{1}=0$ surface (where substance 1 is the principal component of the solid) does not necessarily coincide with the physical surface.

To consider the distance parameters directly, we find that it greatly increases clarity to introduce a minor novelty of notation. It is universally recognized, of course, that in eqn (4), $S^{\sigma}$ and $\Gamma_{i}$ are defined with reference to an arbitrary dividing surface. We now propose to write

$$
-\mathrm{d} \gamma_{\alpha \beta}=S^{\sigma(b)} \mathrm{d} T+\sum_{i} \Gamma_{i}^{(b)} \mathrm{d} \mu_{i}
$$

where " $(b)$ " refers to some objective criterion, $b$, that specifies the reference surface. The best-known of such criteria is, that the surface excess of one component is zero. We further propose that the superscript designating the dividing surface should never be omitted. It is our contention that the reference surface is an indispensable part of the Gibbs equation, and to fail to designate it is to hint at the possibility that it might even be eliminated as a concept. Speaking heuristically, we may refer to the adage, "Out of sight, out of mind."

With reference to the $\Gamma_{i}=0$ surface, the Gibbs equation is written:

$$
-\mathrm{d} \gamma_{\alpha \beta}=S^{\sigma(i)} \mathrm{d} T+\sum_{j \neq i} \Gamma_{j}^{(i)} \mathrm{d} \mu_{j}
$$

$$
-\left[\frac{\partial \gamma_{\alpha \beta}}{\partial \mu_{i}}\right]_{T, P}=\sum_{j \neq i} \Gamma^{(i)}\left[\frac{\partial \mu_{j}}{\partial \mu_{i}}\right]_{T, P} .
$$

Gibbs' discussion of a one-component system, referred to above, can be put in the following form. The two conditions specifying dividing surfaces are: " $t$ ", meaning the surface of tension, and $\Gamma_{1}=0$. Let $\lambda_{1 t}$ be the distance between these two surfaces. Then

$$
\lambda_{1 t}=\frac{\Gamma_{1}^{(t)}}{c_{1}{ }^{\alpha}-c_{1}{ }^{\beta}} .
$$

For a liquid-vapor system appreciably far from its critical temperature, with $\beta$ designating the vapor phase, $c_{i}{ }^{\beta} \ll c_{i}{ }^{\alpha}$,

$$
\lambda_{1 t} \cong \Gamma_{1}^{(t)} / c_{i}^{\alpha} .
$$

Gibbs' conjecture amounts to the hypothesis that $\lambda_{1 t}$ is negligible. For a liquid-solid one-component system, the approximation, eqn (11), cannot be made. However, for such a system, the "surface of tension" does not have the same meaning as it does in a liquid-vapor system. Indeed, it has no operational meaning at all.

To extend this discussion of distances to a binary system, consider Fig. 1. A column, of unit cross section area, extends from $z=0$ to $z=L$. The interface between phases $\alpha$ and $\beta$ lies parallel to the $x y$ plane; the shaded area, in Fig. 1a, indicates the regions in space where an appreciable concentration gradient exists. In Fig. 1b, the gradients of composition are indicated, together with the $\Gamma_{1}=0$ and $\Gamma_{2}=0$ dividing surfaces, which will not in general coincide in space. The distance, $\lambda_{12}$ is obtained as follows. Let the volume of the cylinder be $V$. Since the area of the cylinder is $1 \mathrm{~cm}^{2}$,

$$
\begin{aligned}
V=L & =b+(L-b) \\
& =z_{1}+\left(L-z_{1}\right)=z_{2}+\left(L-z_{2}\right) .
\end{aligned}
$$

Taking the $\Gamma_{1}=0$ surface as the reference surface, we can write for $N_{1}$ (the total number of moles of substance 1 in the column)

$$
N_{1}=z_{1} c_{1}{ }^{\alpha}+\left(L-z_{1}\right) c_{1}{ }^{\beta} \text {. }
$$

With respect to the surfaces at $z_{2}$ and at a general location, $b$, respectively,

$$
\begin{aligned}
N_{1} & =z_{2} c_{1}{ }^{\alpha}+\left(L-z_{2}\right) c_{1}{ }^{\beta}+\Gamma_{1}{ }^{(2)} \\
& =b c_{1}{ }^{\alpha}+(L-b) c_{1}{ }^{\beta}+\Gamma_{1}{ }^{(b)} .
\end{aligned}
$$

For substance 2,

$$
\begin{aligned}
N_{2} & =z_{2} c_{2}{ }^{\alpha}+\left(L-z_{2}\right) c_{2}{ }^{\beta} \\
& =z_{1} c_{2}{ }^{\alpha}+\left(L-z_{1}\right) c_{2}{ }^{\beta}+\Gamma_{2}{ }^{(1)} \\
& =b c_{2}{ }^{\alpha}+(L-b) c_{2}{ }^{\beta}+\Gamma_{2}{ }^{(b)} .
\end{aligned}
$$

With positive sign defined as in Fig. 1, we set

$$
\lambda_{i b} \equiv z_{i}-z_{b}=-\lambda_{b i}
$$

That is, for example,

$$
\lambda_{12}=z_{1}-z_{2}=-\lambda_{21} \text {. }
$$

We designate component 1 as the major component of 
(a)

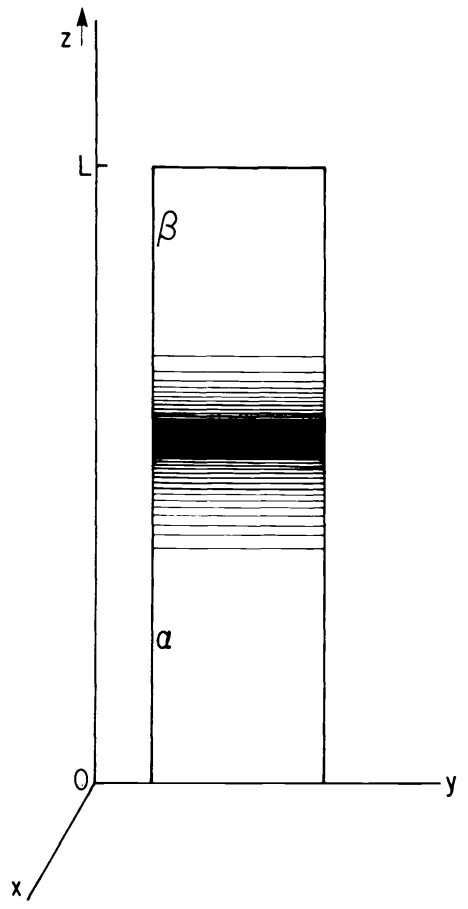

(b)

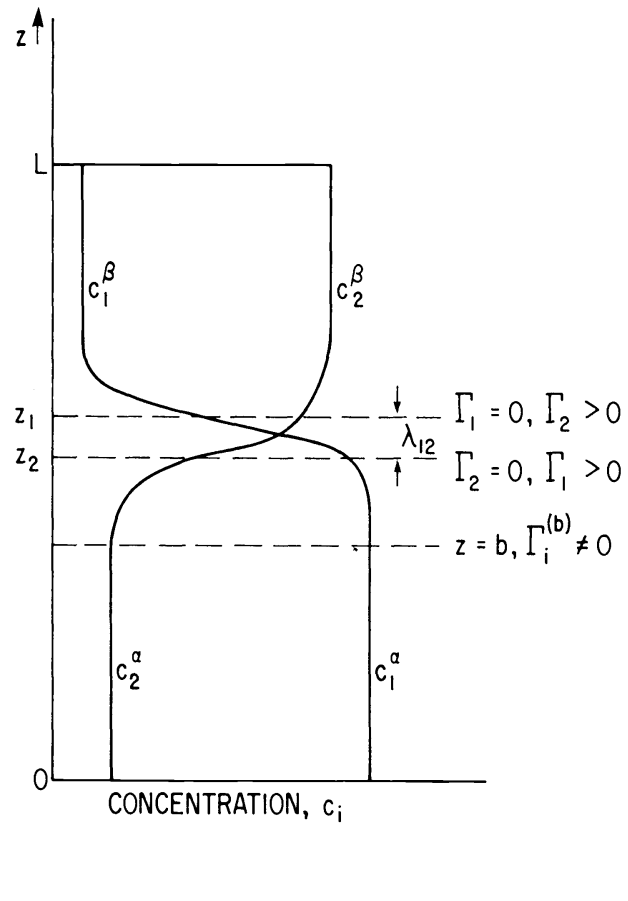

Fig. 1. Concentration profiles and dividing surfaces for a binary, liquid-liquid system.

phase $\alpha$ (the lower phase, in a gravitational field) and we define the concentration differences as

$$
\Delta c_{1} \equiv c_{1}^{\alpha}-c_{1}^{\beta}, \quad \Delta c_{2} \equiv c_{2}^{\alpha}-c_{2}^{\beta} .
$$

Eliminating $N_{1}$ between eqns (13a) and (13b), and $N_{2}$ between eqns (14a) and (14b),

$$
\begin{aligned}
& \Gamma_{1}^{(2)}=\lambda_{12} \Delta c_{1} \\
& \Gamma_{2}^{\left({ }^{(1)}\right.}=\lambda_{21} \Delta c_{2}=-\lambda_{12} \Delta c_{2} .
\end{aligned}
$$

In a similar manner, we obtain

$$
\begin{aligned}
& \Gamma_{1}^{(b)}=\lambda_{1 b} \Delta c_{1} \\
& \Gamma_{2}^{(b)}=\lambda_{2 b} \Delta c_{2} .
\end{aligned}
$$

By further simple manipulation, we obtain

$$
\begin{aligned}
\Gamma_{1}^{(b)} & =\Gamma_{1}^{(2)}+\left(\lambda_{1 b}-\lambda_{12}\right) \Delta c_{1} \\
& =\Gamma_{1}^{(2)}+\lambda_{2 b} \Delta c_{1} \\
\Gamma_{2}{ }^{(b)} & =\Gamma_{2}^{(1)}+\lambda_{1 b} \Delta c_{2} \\
\Gamma_{1}^{(b)} & =\left(\lambda_{12}+\lambda_{2 b}\right) \Delta c_{1} \\
\Gamma_{2}^{(b)} & =\left(\lambda_{21}+\lambda_{1 b}\right) \Delta c_{2} .
\end{aligned}
$$

If we consider a second arbitrary condition $d$ (not one of the $\Gamma=0$ surfaces), we find that

$$
\lambda_{b d}=\frac{\Gamma_{i}^{(b)}-\Gamma_{i}^{(d)}}{\Delta c_{i}} \text { for each } i .
$$

$\dagger$ If this approximation is not made at this point, the derivation is more general but somewhat more lengthy. If the general development is employed, with the low-solubility approximation made at the end, the result is exactly the same as reported here. The more general treatment will be reported elsewhere. ${ }^{8}$
The $\Gamma$ 's can be also expressed in terms of the integrated concentration functions, $c_{1}(z)$ and $c_{2}(z)$. Thus,

$$
\begin{aligned}
\Gamma_{1}^{(1)} & =0=\int_{0}^{L} c_{1}(z) \mathrm{d} z-\int_{0}^{z_{1}} c_{1}{ }^{\alpha} \mathrm{d} z-\int_{z_{1}}^{L} c_{1}{ }^{\beta} \mathrm{d} z \\
& =\int_{0}^{z_{1}}\left[c_{1}(z)-c_{1}{ }^{\alpha}\right] \mathrm{d} z+\int_{z_{1}}^{L}\left[c_{1}(z)-c_{1}{ }^{\beta}\right] \mathrm{d} z \\
\Gamma_{1}^{(b)} & =\int_{0}^{b}\left[c_{1}(z)-c_{1}{ }^{\alpha}\right] \mathrm{d} z+\int_{b}^{L}\left[c_{1}(z)-c_{1}{ }^{\beta}\right] \mathrm{d} z \neq 0 .
\end{aligned}
$$

We may now employ the complete Gibbs-Duhem equations for the two phases, to evaluate $\partial \mu_{1} / \partial \mu_{2}$.

$$
\begin{aligned}
& V^{\alpha} \mathrm{d} P-S^{\alpha} \mathrm{d} T=N_{1}^{\alpha} \mathrm{d} \mu_{1}+N_{2}{ }^{\alpha} \mathrm{d} \mu_{2} \\
& V^{\beta} \mathrm{d} P-S^{\beta} \mathrm{d} T=N_{1}{ }^{\beta} \mathrm{d} \mu_{1}+N_{2}{ }^{\beta} \mathrm{d} \mu_{2} .
\end{aligned}
$$

Here, $V$ and $S$ are the volume and entropy, respectively, of the phases denoted by the superscripts. Dividing by $V^{\alpha}$ and $V^{\beta}$, we obtain

$$
\begin{aligned}
& \mathrm{d} P-S_{v}{ }^{\alpha} \mathrm{d} T=c_{1}{ }^{\alpha} \mathrm{d} \mu_{1}+c_{2}{ }^{\alpha} \mathrm{d} \mu_{2} \\
& \mathrm{~d} P-S_{v}{ }^{\beta} \mathrm{d} T=c_{1}{ }^{\beta} \mathrm{d} \mu_{1}+c_{2}{ }^{\beta} \mathrm{d} \mu_{2}
\end{aligned}
$$

where $S_{v}$ is entropy per unit volume.

When mutual solubilities are low, $\dagger$ i.e. $c_{2}{ }^{\alpha} \ll c_{1}^{\alpha}$, $c_{1}^{\beta} \ll c_{2}^{\beta}$, we may write,

$$
\begin{gathered}
\mathrm{d} P-S_{v}{ }^{\alpha} \mathrm{d} T=c_{1}{ }^{\alpha} \mathrm{d} \mu_{1} \\
\mathrm{~d} P-S_{v}{ }^{\beta} \mathrm{d} T=c_{2}{ }^{\beta} \mathrm{d} \mu_{2} \\
{\left[\frac{\partial \mu_{1}}{\partial P}\right]_{T, \text { sat }}=\frac{1}{c_{1}{ }^{\alpha}}} \\
{\left[\frac{\partial \mu_{2}}{\partial P}\right]_{T, \text { sat }}=\frac{1}{c_{2}{ }^{\beta}} .}
\end{gathered}
$$


Equation (6) can now be put in the form,

$$
\begin{array}{r}
-\left[\frac{\partial \gamma_{\alpha \beta}}{\partial P}\right]_{T, \text { sat }}= \\
=\frac{\Gamma_{1}{ }^{(b)}}{c_{1}{ }^{\alpha}}+\frac{\Gamma_{2}^{(b)}}{c_{2}{ }^{\beta}} \mathrm{e} \\
=\frac{\Gamma_{1}{ }^{(2)}}{c_{1}{ }^{\alpha}}=\frac{\Gamma_{2}{ }^{(1)}}{c_{2}{ }^{\beta}} .
\end{array}
$$

Since, when mutual solubilities are low, the concentration of the major component in moles per unit volume is the inverse of the molar volume $V_{m}$, we have as an excellent approximation,

$$
\begin{aligned}
\Gamma_{1}^{(2)} & =-\frac{1}{V_{m 1}}\left(\frac{\partial \gamma_{\alpha \beta}}{\partial P}\right)_{T, \text { sat }} \\
\Gamma_{2}^{(1)} & =-\frac{1}{V_{m 2}}\left(\frac{\partial \gamma_{\alpha \beta}}{\partial P}\right)_{T, \text { sat. }}
\end{aligned}
$$

Rearranging eqns (17a) and (17b), in the case of low solubility,

$$
\lambda_{12}=\Gamma_{1}^{(2)} / c_{1}{ }^{\alpha}=-\Gamma_{2}^{(1)} / c_{2}{ }^{\beta} .
$$

Combining with eqn (28), we obtain

$$
\lambda_{12}=-\left[\frac{\partial \gamma_{\alpha \beta}}{\partial P}\right]_{T, \text { sat }}
$$

Gibbs $^{1}$ has given a physical interpretation of $\partial \gamma / \partial P$ : it is the change in volume of the system, that accompanies unit increase in interfacial area. A volume divided by an area is, of course, equal to a distance.

\section{THREE-PHASE SYSTEMS}

We have already described the Phase Rule constraints on 3-phase systems. The discussion of the $\lambda$ 's for dividing surfaces can be carried over intact, from binary 2-phase systems, as regards interfaces, to regions far from the lines which bound them. In addition to the surfaces of tension which are associated with a two-phase system, there is a line of tension. ${ }^{9,10}$ If the line of tension is curved, there exists an inward hoop-stress that is the two dimensional analog of the Laplace pressure across a curved interface. ${ }^{11}$ We will, in what follows, restrict the discussion to cases in which the 3-phase line is straight.

Associated with each of the three interfaces in a 3-phase system, there will be one $\Gamma=0$ dividing surface for each component that is present. For a system in which $\theta>0$, the three surfaces of tension must be confluent at the line of tension; but this does not necessarily mean that Gibbsian dividing surfaces are confluent at a line. Rather, they will come together in a region, a volume which lies around a line, which is the analog of the interfacial region between two bulk phases. Somewhere in that volume lies the line of tension. Compare the situation in a twodimensional, two-phase system, where the physical transition occurs over an area which encloses the geometric line of contact.

When we discuss line tension, $\gamma^{l}$, or linear free energy, we mean the excess free energy in the system over that which would be present if the three interfacial free energies (and other surface excess properties) were constant, all the way up to the line of confluence of the interfaces. The excess free energy per unit length of line is analogous to the excess free energy per unit area, the surface free energy.
If in a liquid-liquid-vapor system, no surface active components are present, and if the molecules from liquid 1 adsorb to a negligible extent at the liquid 2-vapor interface, ${ }^{12}$ the confluence of the various $\Gamma_{i}=0$ surfaces will be in the region close to the line of tension, where gradients of local concentrations exist. For each component $i$, the three $\Gamma_{i}=0$ surfaces, extrapolated into the 3-phase region, will in general enclose a triangular prismatic volume. These prismatic volumes for the different components $i, j, \ldots$ will have parallel sides, of course; but they will be displaced, one from another, and will have different cross-sectional areas. If there is appreciable surface activity of any component at any interface, then the $\Gamma=0$ surface for that component will be spatially removed from the corresponding surface of tension (see below) and the lines of intersection of that surface with other $\Gamma=0$ surfaces will be more or less far removed from the region around the line of tension.

In principle, there should be a one-dimensional analog of $\Gamma$, i.e. a linear excess mass, for each component. Let us call it $\Gamma^{l(b)}$, where $(b)$ designates some criterion, just as in eqn (7). For a 2-phase film on a Langmuir trough, this concept can easily be grasped, with respect to the boundaries of the 2-dimensional phases, by analogy to three-dimensional systems.

Some new considerations arise, however, in a 3-phase system with $\theta>0$. For such a system, we can write the linear excess mass $\Gamma_{i}$ with respect to the line of tension: $\Gamma_{i}^{l(t)}$, for each component $i$. In general, these $\Gamma^{l(t)}$ 's will be unequal, and none will be zero. There will exist a $\Gamma_{i}^{l}=0$ line for each component $i$, analogous to the $\Gamma_{i}=0$ surface for a component at an interface. If there are three components, there will be three $\Gamma_{i}^{l}=0$ lines, and they will be at the edges of a triangular prism. (There is no obvious physical significance to the planes that comprise the sides of the prism.) With respect to each $\Gamma_{i}^{l}=0$ line, there will be an excess mass of each other component, $\Gamma_{j}^{l(i)}$. There will also be linear analogs of surface entropy and total energy, for example $S^{l(i)}$ and $U^{l(i)}$.

At present, there do not exist any general, practical methods of measuring line tension. If a time comes when such methods become available, it will be worthwhile to investigate the linear analog of the Gibbs equation.

When one of the phases is solid, the above arguments hold, with the exception that the $\Gamma_{i}=0$ surfaces, extrapolated, will not intersect in such a way as to form a prism. Indeed, the $\Gamma_{i S L}$ and $\Gamma_{i S V}$ surfaces will not, in general, interect at all, if the solid surface is planar (see Fig. 2). The region in Fig. 2a, between the two planar $\Gamma_{1}=0$ surfaces, requires the (novel) formal definition of $\Gamma_{1}$ as being based on the concentration profiles along a plane perpendicular to the solid surface. Then the hump in the $\Gamma_{1}=0$ profile, in the region of the 3 -phase line, corresponds to the fact that $\Gamma_{1}{ }^{l(2)}>0$, for the system sketched.

\section{DISCUSSION OF SURFACE EXCESS FUNCTIONS}

A question may now arise, because of the conventional -used in the Guggenheim-Adam account, ${ }^{5}$ noted in the Introduction. Is not the dependence of $\Gamma$ on arbitrary definitions incompatible with the theory we have just developed, in which $\lambda_{12}$ is a unique quantity? To answer this we note first that the Guggenheim-Adam term, "convention," is ill-chosen, because its common interpretation is the antithesis of "absolute." Actually, however, all the "conventions" $(N, M$, etc.) correspond to what we, here, refer to as " $b$ " conditions: unique specifications for 
(a.)

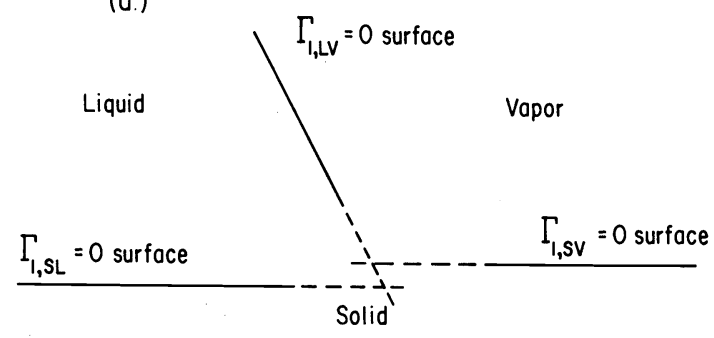

(b.)

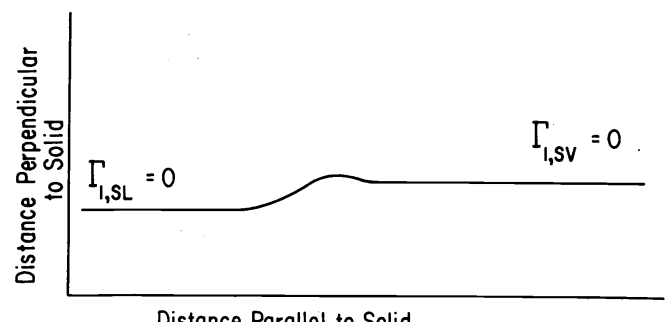

Distance Parallel to Solid

Fig. 2. (a) Dividing $\left(\Gamma_{1}=0\right)$ surfaces in a solid-liquid binary system with a non-zero contact angle, extrapolated as planes. (b) A possible confluence of a $\Gamma_{1, S V}=0$ surface with $a \Gamma_{1, S L}=0$ surface.

geometric surfaces. And the values of any such $\Gamma$ 's can in principle be obtained uniquely as functions of $\Gamma_{1}^{(2)}$ and $\Gamma_{2}{ }^{(1)}$. However, we note in passing that the formulas given by Guggenheim and Adam for computing $\Gamma_{1}{ }^{N}, \Gamma_{1}{ }^{M}$, etc, from $\Gamma_{1}^{(2)}$ or $\Gamma_{2}^{(1)}$ are based on the Gibbs-Duhem equation at constant temperature and pressure,

$$
N_{1} \mathrm{~d} \mu_{1}+N_{2} \mathrm{~d} \mu_{2}=0,
$$

from which the expressions

$$
\begin{aligned}
& \mathrm{d} \gamma / \mathrm{d} \mu_{2}=\Gamma_{2}-N_{2} \Gamma_{1} / N_{1} \\
& \mathrm{~d} \gamma / \mathrm{d} \mu_{1}=\Gamma_{1}-N_{1} \Gamma_{2} / N_{1}
\end{aligned}
$$

are obtained. As already shown, above, this is not a rigorous procedure, and so the significance of the exact values of $\Gamma^{N}, \Gamma^{M}$, etc. reported ${ }^{5}$ is open to re-examination.

As Adamson ${ }^{13}$ has remarked, "The subject... of the various types of surface excess quantities ... has been the source of confusion to both students and research workers." An alleviation of that confusion would be desirable, and so we offer the following discussion and definition.

Gibbs recognized the mathematical possibility of an infinity of dividing surfaces, but he also saw that, physically, one particular specification of a surface was especially important. Mechanologists of Gibbs' day assumed, mathematically, that density and other properties of phases were continuous, up to a geometric surface, and then changed discontinuously. They either considered this discontinuity to be real, or, with Mach, that the question of "reality" was meaningless in this regard. Gibbs saw that, if the description with the discontinuity were taken as describing a reference system, then the difference between the real system and that reference system was operationally meaningful. He also saw that, for a one-component liquid-vapor system, it was possible to locate a surface of discontinuity such that the quantities of matter in the real and the reference systems were the same.

In the usage recommended by IUPAC, ${ }^{14}$ the term, Gibbs dividing surface, refers to any geometrical surface parallel to the interface, which is used to define a reference system. We here propose that the term be restricted to surfaces for which $\Gamma_{i}=0$ for some $i$ th component, in terms of moles; then for any other component, $j$, the term $\Gamma_{j}$ will no longer be used, but instead, either $\Gamma_{j}^{(i)}$, the surface excess with respect to the Gibbs dividing surface for component $i$, or $\Gamma_{j}^{(b)}$, the excess with respect to the surface specified by the criterion, (not convention,) $b$.

In proposing this, we are removing the stultifying conventionalism that appeared to be present in the Guggenheim and Adam treatment, which was noted in the Introduction. We are also alleviating an educational difficulty, which is that novices in surface science often do not realize that, in the Gibbs Equation, the concept of a dividing surface which has an objective location in space is a necessary part of the equation.

In fairness to Guggenheim and Adam, we should remark that what we have done, in the theory given above, could be interpreted as a formal generalization from their multiplicity of $\Gamma$ 's. However, the focus of attention is quite different, in our approach, being not the multiplicity of $\Gamma$ 's, but the infinite number of locations of geometric reference surfaces. From this focussing on the surfaces, we arrive at the existence of an infinity of different $\Gamma$ 's, each having just as much absolute significance, relative to the GDS for that substance, as does a non-zero temperature, relative to the zero of the Kelvin scale.

\section{APPLICATIONS}

We may illustrate the distance parameter concept, using data on Gibbsian absorption of surface active materials such as alcohol and sodium lauryl sulfate, and with the benzene-water system.

(a) Air-water interface. For the ethanol-water system, the maximum value of $\Gamma_{2}^{(1)}$ (where 2 refers to ethanol) is reported to be about $6.5 \times 10^{-10} \mathrm{~mol} / \mathrm{cm}^{2}$ at mole fraction 0.18 . This corresponds to an area of about $25 \AA^{2}$ per ethanol molecule. The corresponding value of $\lambda_{12}$ is $8.8 \AA$.

Using the $\Gamma^{N}$ criterion to specify a dividing surface, the surface excess of ethanol, at 0.18 mole fraction, is $5.5 \times 10^{-10} \mathrm{~mol} / \mathrm{cm}^{2} .5$ The value of $\lambda_{1 N}$ is, then, $7.4 \AA$. The " $N$ " surface is the surface with respect to which the total number of molecules of both species, per unit volume, is the same as in the bulk, all the way up to the dividing surface; and this surface is $7.4 \AA$ from the $\Gamma_{1}=0$ surface. This is illustrative of the computations which could be made, using the various criteria.

For the sodium lauryl sulfate-water system, surface tension data ${ }^{15}$ show that $\Gamma$ is nearly constant, between concentrations of about 2 and $6 \times 10^{-6} \mathrm{~mol} / \mathrm{cm}^{3}$, at about $5.75 \times 10^{-10} \mathrm{~mol} / \mathrm{cm}^{2}$. The value of $\lambda_{12}$, then, varies from $3 \times 10^{4} \AA$, at $2 \times 10^{-6} \mathrm{~mol} / \mathrm{cm}^{3}$, to $1 \times 10^{4} \AA$ at the higher concentration.

It is clear that, for systems containing a surface active component, $\lambda_{12}$ will in general be large, and will give little information about the molecular geometry at the interface. It is easy to recognize why $\lambda_{12}$ will be large. See Fig. 3. The graphical construction for determining the location of the $\Gamma_{2}=0$ surface, by making the two cross-hatched areas equal, shows that the $\Gamma_{2}=0$ surface lies well outside 


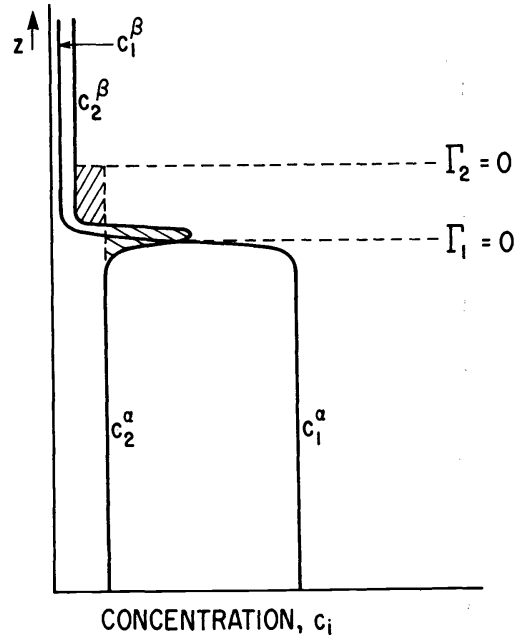

Fig. 3. Concentration profiles and Gibbs dividing surfaces for a binary liquid-vapor system in which one component is surfaceactive.

the region where the concentration gradients of components 1 and 2 are appreciable.

It is also clear that the Gibbs conjecture, that $\lambda_{1 t}$ is an experimentally negligible distance, is not valid with respect to a surface active substance in a multicomponent system. Gibbs made the conjecture with respect to a one-component, liquid-vapor system. It may now be seen that only with respect to non-surface-active components is it applicable to multicomponent systems.

(b) Liquid-liquid systems with no surface active component. For a system such as a hydrocarbon vs water, the concentration profiles should not have peaks such as that in Fig. 3, but monotonic gradients, as in Fig. 1. The two $\Gamma=0$ surfaces must lie in the regions where gradients of concentration exist; and so $\lambda_{12}$ will give some indirect information about the structure of the interface.

The pressure coefficient of interfacial tension has been measured for only a few water-organic systems, and we have found little reliable data available. Perhaps the best data are on benzene vs water; ${ }^{16-18}$ the value, $[\partial \gamma / \partial P]_{T \text {, sat }}=$ -0.0007 ergs per $\mathrm{cm}^{2}$ per atm may be chosen. From this, we compute the values of the surface functions shown in Table 1 . Here $B$ refers to benzene and $W$ to water.

Table 1. Benzene-water system

\begin{tabular}{ll}
\hline$\lambda_{\mathrm{BW}}$ & $0.1 \times 10^{-8} \mathrm{~cm}$ \\
$\left.\Gamma_{\mathrm{W}}{ }^{(B)}\right)$ & $4 \times 10^{-11} \mathrm{~mol} / \mathrm{cm}^{2}$ \\
$\Gamma_{\mathrm{B}}{ }^{(\mathrm{W})}$ & $0.8 \times 10^{-11} \mathrm{~mol} / \mathrm{cm}^{2}$ \\
\hline
\end{tabular}

The small, positive excesses, Table 1, correspond to an excess density of packing of molecules in the interfacial region, over the packing that would be expected on the basis of the bulk densities. It is of interest to examine several possibilities, in assigning that excess to the water or to the benzene. Let us first assume the transition layer to be as sharp as conceivable, and that the water density is constant right up to, but not necessarily including, the last layer of water molecules, and the same for the benzene. A monolayer of water molecules would have approximately $16 \times 10^{-10} \mathrm{~mol} / \mathrm{cm}^{2}$; and the value of $\Gamma_{\mathrm{w}}{ }^{(B)}$ corresponds to an increase of $2.5 \%$ in this concentration per unit area. A monolayer of benzene molecules (assuming an area of $28 \AA^{2}$ per molecule) would contain about $5.9 \times$ $10^{-10} \mathrm{~mol} / \mathrm{cm}^{2}$; and so the value of $\Gamma_{B}{ }^{(\mathrm{W})}$ would correspond to about a $1.3 \%$ increase in packing density. This attribution of all of the excess functions to structural changes in one phase or the other is, of course, an approximation, since it is the interaction that must be considered, to give a complete explanation.

Next, we assume that, in the terminal layer of each phase, the density per unit area corresponds to the bulk values. If the intermolecular distance between water and benzene molecules, across the interface, were the arithmetic mean of the water-water and benzene-benzene distances, which is $(3.10+5.28) / 2=4.19 \AA$, then there should be no change in volume of the system, with increase in interfacial area. However, as Good and Hope ${ }^{15-17}$ have pointed out, an arithmetic mean is an intrinsically unlikely relationship, for intermolecular distances. If a geometric mean is assumed, i.e. $r_{12}=\left(r_{11} r_{22}\right)^{1 / 2}$, then the predicted $r_{12}$ is $4.057 \AA$, which is $3 \%$ below the arithmetic mean. The observed $\lambda_{12}$ corresponds to a $2 \%$ reduction of $r_{12}$ below the arithmetic mean, which is good agreement considering the accuracy of the data. Now, it would be very unlikely for a geometric mean combining rule to be valid unless there was a physical resemblance, between the types of bonds of unlike molecules to each other (i.e. benzene to water) and the bond types of like molecules (i.e. benzene-benzene and water-water.) And such a bond is known to exist, between a hydroxyl and the $\pi$ electrons of benzene. ${ }^{22}$ Its energy has been estimated as about $2.4 \mathrm{kcal} / \mathrm{mol}^{23}$

Thus, considerations of intermolecular potential energy curves will qualitatively account for the value of $\lambda_{12}$. This is, of course, only a first-order calculation, which does not take into account such features as the coordination number of water, nor the possibility of mixing of water and benzene in the transition layer.

\section{SUMMARY}

We have pointed out some consequences of the Phase Rule with respect to adsorption thermodynamics in binary, 2-phase systems and binary or ternary three-phase systems. We have shown that the surface excess functions, in a liquid-liquid binary system, can be obtained from the pressure coefficient of the interfacial tension. From $\partial \gamma / \partial P$, we can also determine the distance, $\lambda_{12}$, between $\Gamma_{1}=0$ surface and the $\Gamma_{2}=0$ surface. We have proposed a removal of the convention of definitions of various surface excess functions, which Guggenheim and Adam had introduced. And we have examined the nature of dividing surfaces in three-phase systems with non-zero contact angles, and in principio, the existence of linear excess thermodynamic functions.

Illustration has been given of the concepts developed, for surface active compounds at the airwater interfaces. In such systems, $\lambda_{12}$ may be very large, and little direct physical interpretation can be given, of such values of $\lambda_{12}$. If surface active components are absent, $\lambda_{12}$ is small, and its value can be used in drawing inferences about interfacial structure. The value of $\lambda_{12}$ for the benzene-water system, approximately $0.1 \AA$, may be interpreted in terms of increased packing density in the terminal layers of either phase, or the closer molecular approach of water molecules to benzene molecules, in comparison to the arithmetic mean combining rule for 
intermolecular distances. Hydrogen bonds between hydroxyls and $\pi$ electron systems are, no doubt, involved.

Acknowledgement-This paper summarizes work that was supported by the National Science Foundation under grants No. GK 382, GK 1985 and ENG-75-03479. The author expresses thanks to Dr. F. P. Buff, for discussions of the concept of interfacial distance parameters. The fruits of these discussions with respect to systems where solubility is appreciable, and with regard to energy and entropy considerations, will be presented in future publications.

\section{REFERENCES}

${ }^{1}$ J. W. Gibbs, Collected Works. Vol. 1. Dover, New York (1961).

${ }^{2 a}$ F. P. Buff, 38th National Colloid Symposium, Austin, Texas (1964). ${ }^{2 b}$ F. P. Buff and R. A. Lovett, in Simple, Dense Fluids (edited by H. L. Frisch and Z. W. Saltzburg), Academic Press, New York (1968). ${ }^{2 c}$ An authorized review of Butt's thermodynamic theory for one-component systems has been given by J. C. Melrose, Ind. Eng. Chem. 60, 53 (1968).

${ }^{3}$ R. Lovett, P. W. DeHaven, J. Vieceli and F. P. Buff, J. Chem. Phys. 58, 1880 (1973).

${ }^{4}$ F. P. Buff, The theory of capillarity, in Handbuch der Physik. (edited by S. Flugge) Vol. 10. Springer, Berlin (1960).

${ }^{5}$ E. A. Guggenheim and N. K. Adam, Proc. Roy. Soc. (Lond.), A139, 218 (1933).
${ }^{6}$ R. Defay, I. Prigogine, A Bellemans and D. H. Everett, Surface Tension and Adsorption. Wiley, New York (1966).

${ }^{7}$ E. A. Guggenheim, Thermodynamics. 4th Edn, North Holland, Amsterdam (1954).

${ }^{8}$ R. J. Good and F. P. Buff, A preliminary report of the general treatment was given by R. J. Good and F. P. Buff, 43rd National Colloid Symposium, Cleveland (1969). To be published.

'J. E. Lennard-Jones, Proc. Phys. Soc. 213, 461 (1931).

${ }^{10}$ F. P. Buff and H. Saltzburg, J. Chem. Phys. 26, 23 (1957).

${ }^{11}$ N. L. Gershfeld and R. J. Good, J. Theoret. Biol. 17, 246 (1967).

${ }^{12}$ R. J. Good, J. Colloid Interface Sci. 52, 308 (1975).

${ }^{13}$ A. W. Adamson, Physical Chemistry of Surfaces. 2nd Edn, Wiley Interscience, New York (1967).

${ }^{14}$ Definitions, Terminology and Symbols in Colloid and Surface Chemistry. IUPAC Council, Washington, D.C. (1971); Butterworth, London (1972).

${ }^{15}$ A. P. Brady, J. Phys. Colloid Chem. 53, 56 (1949).

${ }^{16}$ A. S. Michaels and E. A. Hauser, J. Phys. Chem. 55, 408 (1951).

${ }^{17}$ M. E. Hassan, R. F. Nielsen and J. C. Calhoun, Trans. AIME 192, 57 (1951).

${ }^{18} \mathrm{H}$. Y. Jennings, J. Phys. Chem. 62, 323 (1967).

${ }^{19}$ R. J. Good and C. J. Hope, J. Chem. Phys. 53, 540 (1970).

${ }^{20}$ R. J. Good and C. J. Hope, J. Chem. Phys. 55, 111 (1971).

${ }^{21}$ R. J. Good and C. J. Hope, J. Colloid Interface Sci. 35, 171 (1971).

${ }^{22} \mathrm{~J}$. A. Pople, Hydrogen Bonding (edited by D. Hadzi and H. W. Thompson), Pergamon Press, London (1959).

${ }^{23}$ R. J. Good and E. Elbing, Ind. Eng. Chem. 62(3), 54 (1970). 